\title{
Trabajo decente desde los lineamientos de la OIT: aplicabilidad en el sector agrícola cebollero de Aquitania Boyacá
}

\section{Decent work from the ILO guidelines: applicability in the Aquitania Boyacá agricultural sector}

\author{
DOI: https://doi.org/10.17981/juridcuc.16.1.2020.04
}

Fecha de Recepción: 25/10/2019. Fecha de Aceptación: 03/12/2019

\author{
Hugo Armando Ramírez Acevedo \\ Universidad de Boyacá (Colombia) \\ hugramirez@uniboyaca.edu.co
}

\section{Néstor Enrique Ulloa Corredor}

Universidad de Boyacá (Colombia)

neulloa@uniboyaca.edu.co

Para citar este artículo:

Ramírez, H. y Ulloa, N. (2020). Trabajo decente desde los lineamientos de la OIT: aplicabilidad en el sector agrícola cebollero de Aquitania Boyacá. JURÍDICAS CUC, 16(1). 97-126. DOI: http://dx.doi.org/10.17981/juridcuc.16.1.2020.04

\section{Resumen}

El concepto de "trabajo decente" es un elemento esencial en los ordenamientos jurídicos de los Estados democráticos y aún más de aquellos que hacen parte de la Organización Internacional del Trabajo (OIT). Así mismo, la obligación que contraen los Estados miembro respecto a la garantía de los derechos laborales desde el contexto del trabajo decente, debe verse reflejada en una acción efectiva de protección aún más en el sector agrícola. Desde esta lógica, el objetivo planteado conlleva a efectuar un análisis sobre "el trabajo decente desde los lineamientos de la OIT y su aplicabilidad en el sector agrícola cebollero del municipio de AquitaniaBoyacá”. Investigación desarrollada desde un enfoque cualitativo, el cual permitió interactuar con la muestra poblacional. Así mismo, fue necesario elegir 46 trabajadores del sector agrícola, en 5 empresas comercializadoras de cebolla larga, cada una con un promedio de 13 trabajadores, en las cuales se practicó una entrevista semiestructurada, estableciendo categorías y subcategorías que permitieron concretar la información, además del análisis y la interpretación de las fuentes tanto primarias como secundarias que se tuvieron en cuenta para tal fin. El resultado de este proceso, ha permitido inferir que los trabajadores del sector agrícola adolecen de la protección del Estado en materia laboral y menos aún desde la garantía que otorga el convenio con la OIT en orden a la consecución de un trabajo decente. Por tanto es importante repensar y reestructurar la metodología normativa respecto a su aplicación con el fin de garantizar los derechos laborales a dicha población. Palabras clave: derechos laborales; Organización Internacional del Trabajo; prestaciones sociales; protección estatal; trabajo decente; trabajador rural

\section{Abstract}

The concept of "decent work" is an essential element in the legal systems of democratic States and even more so of those that are part of the International Labour Organization (ILO). Likewise, the obligation of the Member States to guarantee labour rights in the context of decent work must be reflected in effective action to protect it even more in the agricultural sector. From this logic, the proposed objective leads to an analysis of "decent work from the guidelines of the ILO and its applicability in the agricultural sector of the municipality of AquitaineBoyacá". Research developed from a qualitative approach, which allowed interaction with the population sample. Likewise, it was necessary to choose 46 workers from the agricultural sector, in 5 companies marketing long onions, each with an average of 13 workers, in which a semi-structured interview was carried out, establishing categories and subcategories that allowed the information to be specified, in addition to the analysis and interpretation of both primary and secondary sources that were taken into account for this purpose. The result of this process has allowed us to infer that workers in the agricultural sector suffer from the protection of the State in labor matters, and even less from the guarantee that the convention with the ILO provides in order to achieve decent work. Therefore, it is important to rethink and restructure the normative methodology regarding its application in order to guarantee labor rights to this population.

Keywords: Labor rights: International Labor Organization; social benefits; state protection; decent work; rural worker 


\section{INTRODUCCIÓN}

Desde que Juan Somavia, director general de la Organización Internacional del Trabajo, en desarrollo de las memorias que presentara ante la Conferencia Internacional del Trabajo (CIT, 2019), introdujera el concepto de trabajo decente como aquella actividad productiva justamente remunerada, que se desarrolla en condiciones de equidad, libertad, seguridad y respeto por la dignidad de la persona humana, la OIT ha desarrollado un plan de acción con el fin de materializar el concepto de trabajo decente como un criterio estandarizado que rija en todas las relaciones de trabajo en el mundo y a la vez se emplee como una respuesta a los nuevos modelos de producción que entronizan la desigualdad y precarizan el empleo, sustituyéndolos por una noción que reivindica la importancia del trabajo y lo ubica como uno de los criterios más importantes para el desarrollo humano.

Para el cumplimiento de ese propósito la Organización Internacional del Trabajo-OIT ha dotado de alcance y sentido al concepto a través de cuatro objetivos fundamentales, los cuales Blanco (2002) describe de la siguiente forma:

Promoción de la Declaración de la OIT relativa a los principios y derechos fundamentales en el trabajo de junio de 1998 por medio de la cual la Conferencia Internacional del Trabajo reafirmó su adhesión a los ideales fundacionales de la OIT relativos a los principios y derechos fundamentales en el trabajo. 2. Promoción del empleo e ingresos decentes que constituye, al decir de la OIT, el corazón mismo de su misión (...) 3. Fortalecimiento de la protección y de la seguridad social para todos que, en últimas, puedan atender las necesidades esenciales de supervivencia humana (...) 4. Fortalecimiento y apoyo del tripartismo y del diálogo social equilibrado (p. 3).

Así es como el concepto de trabajo decente se ha convertido en una hoja de ruta para la construcción de políticas, normas y actuaciones, a la vez que condensan los propósitos de la OIT a lo largo de la historia, permitiendo plantear una alternativa a futuro frente al deterioro de las relaciones laborales, la creciente desigualdad, dis- 
criminación y aumento de la pobreza, al punto que se ha convertido en uno de los criterios del Plan de Desarrollo Sostenible 2030 de la Organización de Naciones Unidas.

La dimensión integradora y principialistica del axioma de trabajo decente se erige en una declaración de intenciones para la unificación de los parámetros mundiales de generación de condiciones laborales, a la vez que en sí mismo se convierte en un diagnóstico del entorno de trabajo que permite verificar el déficit de condiciones laborales de manera particular.

La medición del trabajo decente permite conocer las condiciones sociales en las que se prestan los servicios y formular, a partir de la obtención de datos empíricos, políticas y diálogos tendientes a la superación de las condiciones de precariedad en el empleo.

El sector rural colombiano se caracteriza por tener unas condiciones laborales poco seguras, con altas tasas de trabajo informal, desigualdad en las condiciones de acceso a la tierra, una desprotección estatal de carácter histórico, falta de afiliación y cobertura a los sistemas de seguridad social, falta de estabilidad, baja remuneración y un desconocimiento de los derechos constitucionales y legales contemplados en el ordenamiento laboral.

El trabajo en el sector rural afronta grandes retos como las altas tasas de informalidad, la desigualdad en el uso y acceso a la tierra, la desprotección estatal, la poca estabilidad, la mala remuneración y el desconocimiento de los derechos laborales.

En este trabajo de investigación, se analizan las condiciones de empleo bajo los componentes conceptuales y jurídicos de la categoría de trabajo decente en el sector agrícola cebollero del municipio de Aquitania-Boyacá, y desde este contexto, y teniendo en cuenta los instrumentos de medición de esta categoría conceptual, determinar si ellos se observan en la actividad desplegada por los trabajadores que ejercen el oficio de producción de cebolla en dicho municipio, a través de un baremo de medición de las condiciones particulares y generales de quienes prestan sus servicios en esta actividad productiva. 


\section{Desarrollo}

A continuación, se desarrollan tres aspectos relativos a: 1) La fundamentación conceptual y jurídica desde la óptica de la OIT en relación al trabajo decente y su aplicabilidad en la muestra poblacional. 2) Caracterización y condiciones laborales actuales de la muestra poblacional y 3) Aplicabilidad de las prerrogativas establecidas en los planteamientos de la OIT, relacionados con el trabajo decente en dicha población. Para lograr tal fin, se han establecido categorías de análisis evidenciadas en cada una de las gráficas las cuales responden a cada uno de los aspectos antes referenciados mediante su interpretación y análisis.

Fundamentos conceptuales y juridicos previstos por la O.I.T, relativos al "trabajo decente" y su perspectiva en el sector agricola cebollero del Aquitania - Boyacá.

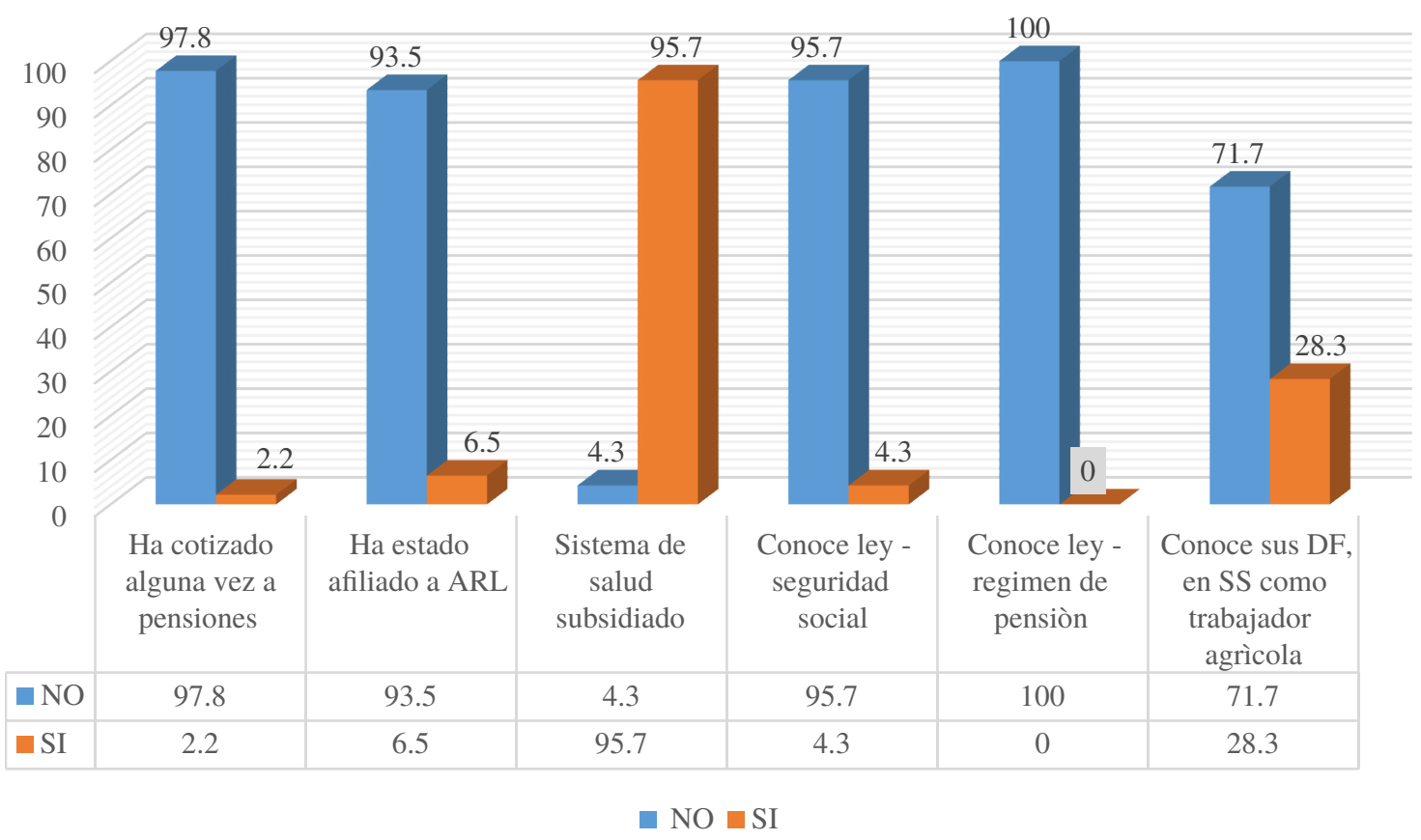

Figura 1. Análisis de la categoría Entorno Laboral. Síntesis de datos surgidos de la muestra poblacional correspondiente a 46 personas de 5 empresas comercializadoras de cebolla larga.

Fuente: Elaboración propia con base en la información de las entrevistas practicadas a la población. 
El primer aspecto denominado "entorno laboral" descrito en la Figura 1, permite visualizar tópicos particulares sobre los cuales se desarrolla el entorno laboral de los trabajadores del sector agrícola en las empresas comercializadoras de cebolla larga del municipio de Aquitania-Boyacá. Percepción que se analizará desde el contexto de trabajo decente propuesto por la Organización Internacional del Trabajo.

Atendiendo al contenido de la Figura 1 y desde un análisis deductivo, permite inferir que el total de la población entrevistada desconoce el contenido jurídico referido al régimen de pensión, por lo cual un amplio porcentaje de ellos no han cotizado a dicho régimen.

De igual forma, la mayoría de trabajadores de este sector agrícola desconoce lo normado sobre el Sistema General de Seguridad Social Integral, es decir aquel que "protege al ser humano de manera integral contra las necesidades sociales (...) y que además tiene por objeto "garantizar los derechos irrenunciables de la persona y la comunidad para obtener la calidad de vida acorde con la dignidad humana, mediante la protección de las contingencias que le afecten" (Legis, 2018, p. 20). De esta forma, tanto el desconocimiento de la norma como la falta de cotización al Sistema de Seguridad Social, no solo conlleva al deterioro del mínimo vital, sino también conduce a crear marginalidad presente y futura.

Al sistema subsidiado de salud, están afiliados la mayoría de los trabajadores del sector laboral que son sujetos de esta investigación, por lo cual no conciben la necesidad de pagar el porcentaje correspondiente en caso de vínculo contractual y menos aún de exigir al empleador pagar dichos conceptos, pues entienden que el Estado es quien debe asumir estas cargas independientemente si están laborando de manera formal o informal. De tal forma que, si el empleador quiere formalizar un contrato con sus trabajadores seguramente encontrará reticencia por parte de ellos, en el entendido que no admiten descuentos de su salario. 
Por su parte el contenido de la Ley 100 (art. 212, 1993), entiende que el régimen subsidiado tiene como propósito "financiar la atención en salud a las personas pobres y vulnerables y sus grupos familiares que no tienen capacidad de cotizar, para integrarlos al sistema de salud". En este contexto, aunque el Estado garantiza los derechos de los trabajadores del sector agrícola en materia de salud, surge como consecuencia para el sistema un desequilibrio económico, al no percibir los porcentajes de cotización debidos por las partes en la relación laboral o de forma independiente.

Según Cortez, (2016), la "financiación de este régimen se da por esquema de subsidio a la demanda, lo que implica identificar a los beneficiarios a través de la encuesta del Sisbén y se ejecuta por medio de una cotización subsidiada total o parcialmente, que compromete recursos fiscales y parafiscales" (p. 328).

Con todo, la población intervenida formalizada tiene las condiciones para realizar sus respectivas cotizaciones al sistema y de exigir al empleador el pago de las mismas. Sin embargo, no es la costumbre y por tanto prefieren el salario pleno a tener que soportar algún descuento en salud o pensión.

La afiliación de los trabajadores del sector agrícola en cuestión al sistema general de riesgos laborales es muy deficiente, pues como muestra la Figura 1, la mayoría no están afiliados, lo cual indica que están expuestos a soportar sobre si mismos cualquier calamidad en relación con el desarrollo de su trabajo. El sistema está estructurado con el fin de garantizar la integridad del trabajador ante cualquier riesgo, tal como lo define Puyana (2013):

En la actividad laboral el trabajador se halla sometido a una serie de contingencias derivadas del trabajo que potencialmente pueden causarle daños al organismo e inclusive la muerte. Así, los riesgos están latentes dentro de la zona de trabajo, comunes a todos los trabajadores o individuales para un trabajador originados por los elementos utilizados en el trabajo o surgidos del lugar del trabajo y de los que no puede sustraerse sin ninguna estructura de prevención y protección que los elimine o por lo menos aminore el impacto sobre su organismo (p. 15). 
En este mismo sentido la Ley 1562 (2012), por la cual se "modifica el Sistema de Riesgos Laborales, establece que el sistema está destinado a prevenir, proteger y atender a los trabajadores de los efectos de enfermedades y accidentes que puedan ocurrirles con ocasión o como consecuencia del trabajo que desarrollan". De igual forma se relaciona la capacitación que desde el ámbito de salud ocupacional se debe realizar en la empresa con el fin de prevenir accidentes de trabajo, enfermedades de tipo laboral y además mejorar las condiciones de trabajo entre otras.

En este orden de ideas y en relación con el factor poblacional sujeto de la investigación es claro el respaldo jurídico para este sector. No obstante, la no afiliación ya sea por parte del empleador o de forma independiente, acarrea consecuencias funestas para el trabajador que, en caso de calamidad, no tendrá el reconocimiento de sus prestaciones correspondientes. Situación muy común en los trabajadores del sector agrícola y que finalmente lleva concluir que lo dispuesto en el cuerpo jurídico del Estado respecto a los derechos laborales no son eficaces en su aplicación y por ende se desvirtúa lo establecido por la OIT, respecto a la garantía de un "trabajo decente".

Ahora bien, atendiendo al objeto epistemológico de la OIT sobre el mejoramiento de las condiciones integrales del asalariado, considera que "existen condiciones de trabajo que implican injusticia, miseria y privaciones, lo que a su vez origina tal descontento que la paz y la armonía universales están en peligro y es urgente mejorar esas condiciones (...) (Campos, 2017, p. 84).

Así mismo, la OIT en 2008, mediante el documento de medición del trabajo decente, alude a cuatro objetivos estratégicos: "derechos en el trabajo, oportunidades de empleo, protección social y el diálogo social. Cada uno de ellos cumple, además, una función en el logro de metas más amplias como la inclusión social, erradicación de la pobreza, fortalecimiento de la democracia, desarrollo integral y la realización personal" 
(OIT, 2008, p. 6). De igual forma, el objetivo No. 8 de la Agenda 2030 sobre desarrollo sostenible desde la óptica de trabajo decente invita a:

Promover el crecimiento económico inclusivo y sostenible, el empleo y el trabajo decente para todos,(...), el trabajo decente es a la vez un medio y un fin en la consecución del desarrollo sostenible, establece el compromiso de fomentar un sector empresarial dinámico, de proteger los derechos laborales y de cumplir los requisitos sanitarios y ambientales de conformidad con los acuerdos pertinentes, como las normas de la OIT y los Principios Rectores sobre las Empresas y los Derechos Humanos definidos por las Naciones Unidas (...) (CIT 2019, p. 14).

En este sentido el Estado colombiano ha incorporado en la normativa interna los lineamientos de la OIT respecto al desarrollo de objetivos que conduzcan al desarrollo del trabajo decente. "La OIT tiene una Constitución, que es un instrumento de derecho internacional, obligatorio para Colombia, por ser miembro y haberla ratificado mediante Ley 49 de 1919" (Villegas, 2016, p. 4). Desde esta lógica, se puede concluir que todo trabajador que desarrolle su labor cualquiera que esta sea, está protegido por las normas tanto del derecho internacional como del derecho interno de tal forma que, infringir dichos preceptos conducirá a crear un ámbito de desconfianza por parte de los órganos internacionales expertos en la materia y sobretodo se evidenciará el detrimento de los derechos fundamentales.

Lo anterior indica que los trabajadores del sector agrícola mencionado están al margen de las prerrogativas tanto de la OIT como de la normativa interna pues como se ha dicho, la aplicación de la norma no es eficaz y lo que se busca es el quiebre de la misma con el fin de obtener algún tipo de beneficio empresarial o individual, dado lo cual se lesionan también derechos fundamentales contenidos en la Constitución Política y en los Tratados Internacionales relativos a la materia. 
Caracterización y las condiciones laborales actuales de los trabajadores del sector agrícola cebollero de Aquitania - Boyacá

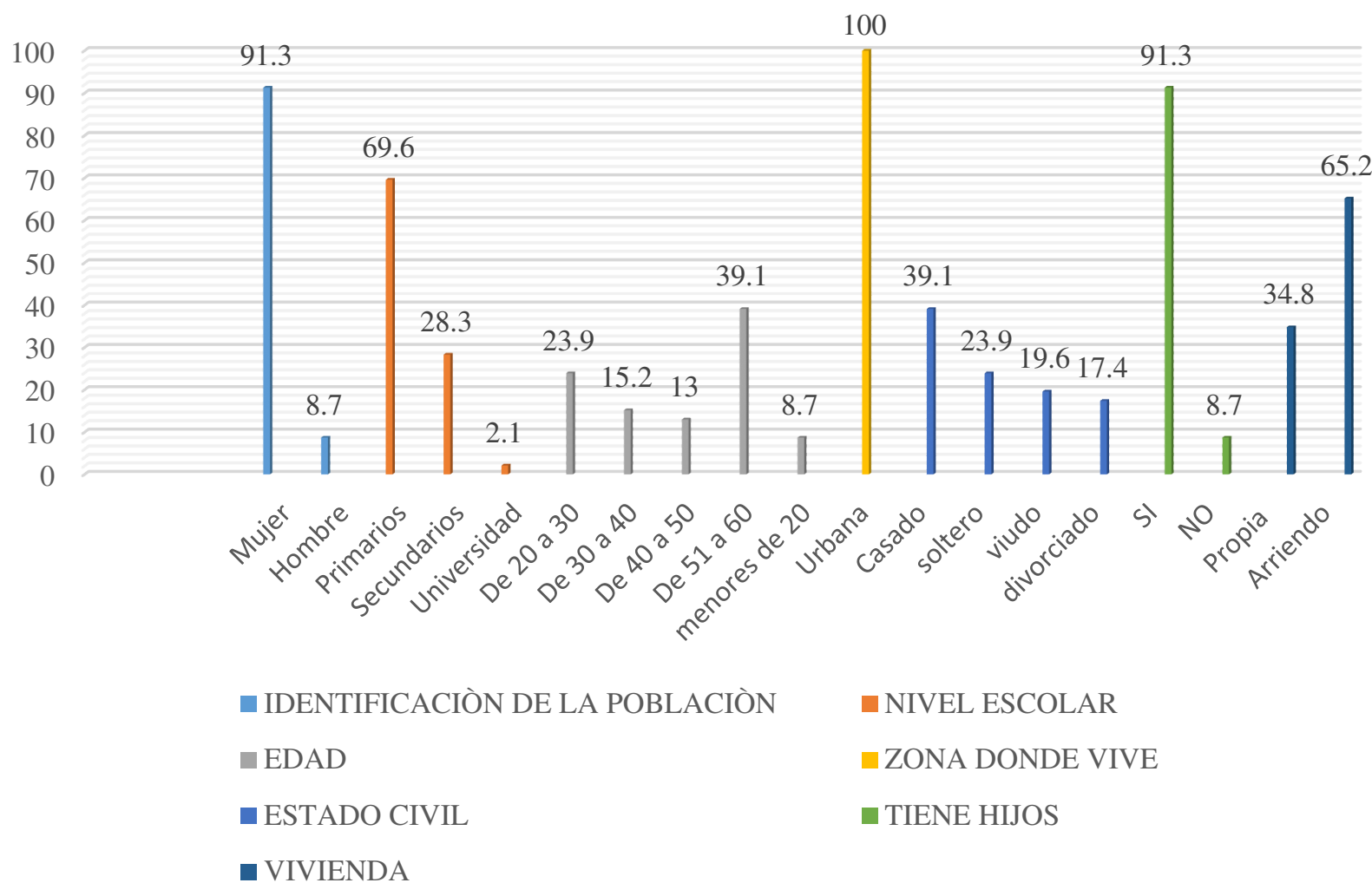

Figura 2. Condiciones particulares, familiares, laborales. Fuente: Elaboración propia con base en la información de las entrevistas practicadas a la población.

Un segundo aspecto, respecto a la caracterización y las condiciones laborales actuales de los trabajadores del sector agrícola cebollero de Aquitania-Boyacá, según los datos arrojados especificados en la Figura 2, permite visualizar como la mayoría de la población que desempeña este tipo de oficio son mujeres, la mayoría hacen parte de la tercera edad, las cuales tienen un nivel de formación básica primaria. Más de la mitad de la población intervenida no posee casa propia y viven en arriendo, todos en la zona urbana. De igual forma, la mayoría de la población tienen hijos nacidos en hogares debidamente conformados, pero también alcanza un alto margen los hijos habidos en hogares disfuncionales o monoparentales. 
Otra caracterización importante que evidenciamos en la Figura 2, está relacionada con la poca intervención del hombre en esta actividad. De igual forma, más de la mitad de la población es mayor de 30 años y en porcentaje reducido esta la población joven, los cuales académicamente han terminado estudios secundarios.

Desde un contexto analítico dicha caracterización permite inferir como a medida que va cambiando el contexto social también van cambiando las formas de relación social en todo sentido. Es decir, la evolución social conlleva necesariamente a una evolución antropológica la cual va adaptándose a ese conglomerado de elementos globalizados tanto económicos, tecnológicos como psicosociales y deconstructivos que permean de alguna forma las constantes ya establecidas y marcadas por el tiempo y la costumbre. Perspectiva compatible con lo planteado por (Perroux, citado por Roth, 2004), quien "definía el desarrollo como la combinación de cambios mentales y sociales de una población, con factores de crecimiento, acumulación y producto global. Los problemas del desarrollo implican preocupaciones económicas, sociales, políticas, técnicas y psicológicas, pero en primera instancia son problemas humanos" (p. 2).

También se "identifica el cambio social con la noción de progreso entendida como evolución sociocultural" (Escalante \& Miñano, citado por Roth, 2004), la cual conduce a percibir los fenómenos sociales desde el contexto globalizado, mercantilista y cuyo fin es el fortalecimiento de la producción y el capital.

$\mathrm{Al}$ respecto el autor citado establece que:

El término evolución sociocultural de uso antropológico, hace referencia a las variaciones innovativas efectuadas en el marco de una cultura. Se trataría de un mecanismo activo para el mejoramiento social que afecta su estructura y funcionamiento merced a la variedad y frecuencia de intercambios que se establecen entre los elementos de la cultura con otros de carácter foráneo, en lo que se conoce como difusión (Roth, 2004, p. 3). 
En este contexto de desarrollo antropológico y sociocultural es que debemos comprender nuestro sistema jurídico, el cual, es reflejo fiel del estado en que se encuentra una sociedad. Si el sistema jurídico esta direccionado hacia fines constitucionales, seguramente el amparo estatal respecto a derechos fundamentales se verá reflejado en la calidad de vida de cada uno de sus habitantes. Por el contrario, si el sistema jurídico se enfoca hacia fines economicistas entonces las consecuencias serán óptimas para el Estado, pero deficientes para asegurar la calidad de vida de los administrados, fortaleciéndose de esta forma el carácter privatista estatal. Situación determinada por las condiciones neoliberales, "que llevan a concepciones diferentes acerca de las garantías y beneficios consagrados en la Constitución a favor de trabajadores y empleadores" (Romero, 2011, p. 294).

En esta línea de reflexión, se ubica el derecho laboral colombiano de los últimos años, donde aspectos como la tercerización, flexibilización laboral, cooperativas y empresas de trabajo asociado son el resultado de la privatización de donde surgen tanto empresas públicas como privadas como intermediarias en la relación laboral y las cuales tienen el control en cuanto a la contratación del personal y oferta de puestos de trabajo.

Situación que se vivencia en todo tipo de relación laboral, pues se amplía el margen de flexibilización laboral que se sustenta en el ordenamiento jurídico como por ejemplo en la Ley 789 (2002), que tiene como línea esencial ajustar las condiciones de empleo al mundo globalizado creando beneficios para las grandes empresas y precarizando las condiciones laborales. Pensamiento que se refleja también en Rodríguez (2007), el cual hace notar el detrimento de los derechos laborales en aras de favorecer o estar en línea con el mundo económico y globalizado¹. De igual forma la Ley 50 (1990)

\footnotetext{
${ }^{1}$ El mismo autor además firma que la Ley 789 (2002), es una parte primordial de la política de flexibilización laboral y está relacionada con las tendencias generadas por la globalización y la firme internacionalización de la economía, la cual implantó cambios importantes en las relaciones laborales, al hacer una reducción de las indemnizaciones por despido injusto, reducir el pago de horas extras y disminuir los recargos por festivos y dominicales.
} 
que se constituye según Isaza (2003), en el "primer paso en la flexibilización al mercado laboral en Colombia, la cual estuvo orientada a facilitar la creación de nuevos empleos porque hizo menos costosa la contratación y los despidos de los trabajadores" (p. 107). En el mismo sentido se concibe la Ley 1429 (2010) sobre formalización y generación de empleo, que otorga beneficios a las empresas que contraten a personal con características especiales. Sin embargo, este tema visto desde un ángulo analítico más profundo conlleva a la precarización salarial, tal como lo evidencia Rojas (2016) al concluir que la "flexibilización laboral en lugar de crear empleo ha traído más informalidad y por tanto disminución de las garantías prestacionales" (p. 17). Desde este contexto finalmente y siguiendo a Ostau de Lafont de León (2015), sostiene que;

El neoliberalismo propone flexibilizar los mercados, particularmente el del trabajo. 1. Las políticas de regulación de las relaciones laborales eliminan la protección del trabajador, conservan las normas de garantías mínimas y procuran la individualización de las relaciones laborales (deslaboralización). Los procesos de regulación laboral y, en general, de flexibilización suponen la reapertura de la autonomía privada como elemento configurador del contrato de trabajo y de las relaciones en la empresa, para ajustar la regulación a la realidad de un mercado de trabajo en el que se ha ido produciendo la ruptura del modelo anterior. La consecuencia de esto es que vuelve a recuperarse el discurso civil de la libertad contractual en detrimento de la regulación colectiva (p. 7).

En términos generales, desde estos lineamientos teóricos se puede establecer que las condiciones laborales actuales de los trabajadores de las empresas comercializadoras de cebolla larga de Aquitania-Boyacá, operan desde este contexto globalizado ya sea porque conocen su metodología jurídica o porque el trabajo agrícola es parte de la costumbre campesina en la cual no figuran prestaciones de ningún tipo por el desarrollo del trabajo. Como se infiere de la Figura 2, la mayoría de la población entrevistada son mujeres con estudios básicos y además se muestra un alto índice de madres cabeza de familia con un promedio de edad mayoritaria de 30 años. 
Lo anterior significa que las condiciones particulares de los trabajadores en cuestión, se ven permeadas por la desintegración familiar, la autonomía de la mujer, los contextos de infidelidad, etc. Algo muy común en la última década, por lo cual, la mujer tiene que desempeñar este tipo de labores en aras de su manutención e igualmente la de sus hijos, pues en algunos casos, el conyugue no se responsabiliza y no coadyuva en su sostenimiento, y en otros aporta una cuota mínima impuesta por vía judicial, no logrando cubrir el mínimo vital de los menores, razón por la cual las trabajadoras del sector se ven forzadas a laborar bajo cualquier condición, situación aprovechada por las empresas pagándoles un porcentaje mínimo de acuerdo a lo que produzcan y sin ninguna garantía de sus derechos prestacionales.

Desde el contexto familiar, una característica evidentemente importante, es que la mayoría de la población intervenida tiene hijos y vive en casas arrendadas, circunstancia que refuerza la obligatoriedad de desempeñar la labor, aceptando las condiciones propuestas por el empleador y menos aún acudir a la jurisdicción laboral para hacer efectivo el reconocimiento de sus derechos prestacionales, pues al tratarse de un pueblo pequeño surge el miedo en el trabajador que si reclama por vía judicial, lo que sigue es la no contratación en otra empresa.

De esta forma, el ámbito laboral de la población intervenida se ve vulnerada en cuanto al progreso tanto personal como familiar, pues no se evidencia garantía de estabilidad laboral, -esta última entendida como aquella garantía de permanencia o duración de una persona en su labor - (Solano, 2019, p. 50), mejora de salarios, promoción o ius variandi positivo y menos aún el reconocimiento de prestaciones sociales lo que lleva a constatar que lo dispuesto en el contenido normativo laboral sobre trabajo decente no se aplica desde esta perspectiva y en esta realidad.

Continuando en esta misma línea de reflexión, la Figura 3, en orden de porcentaje descendente, refleja como la mayoría de los trabajadores son dependientes, es decir, están supeditados a las exigencias de su empleador de forma constante y sin tener la posibilidad de desempeñar otro oficio quizá por la limitación del tiempo o por la complejidad de sus labores. 


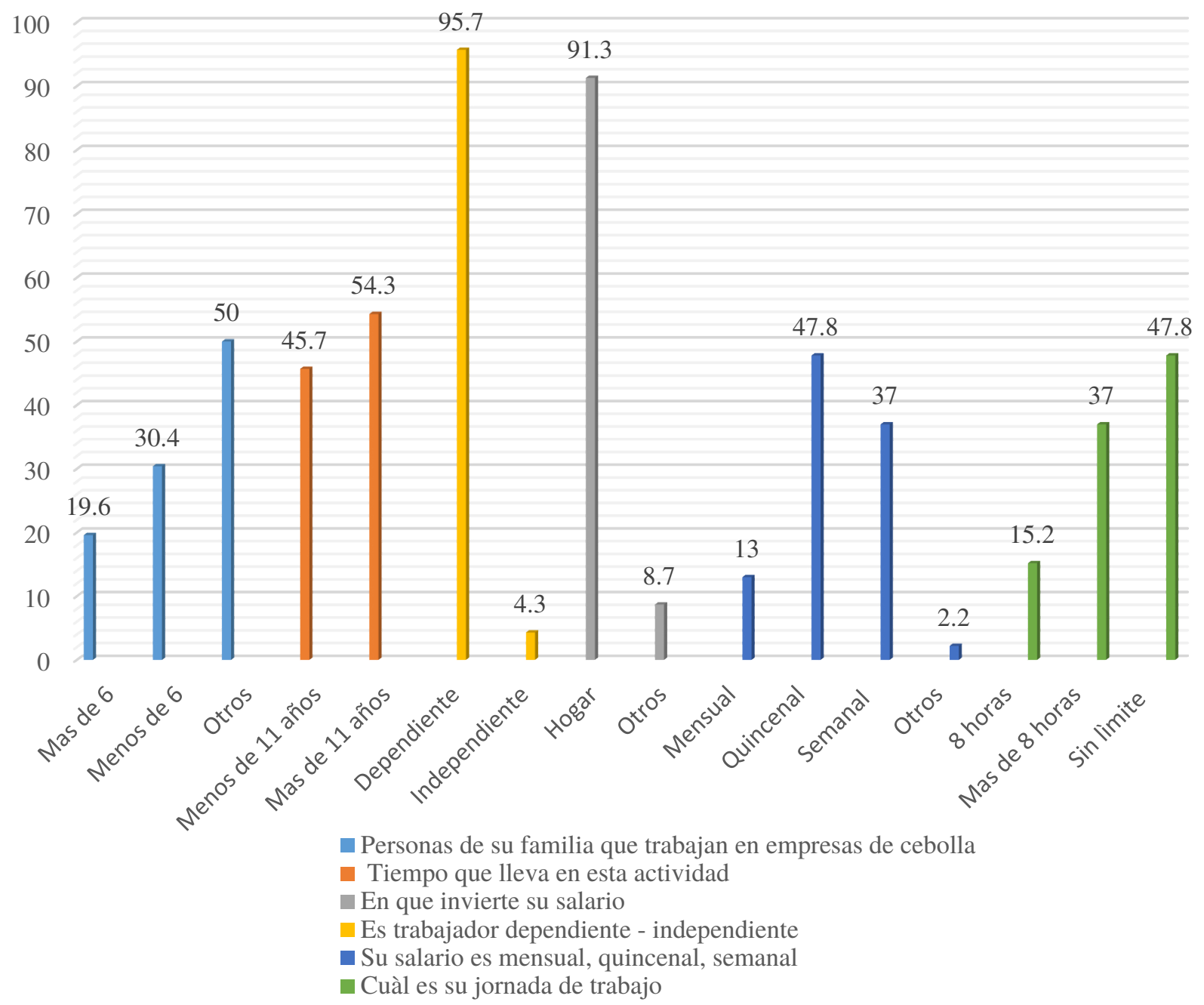

Figura 3. Condiciones particulares, familiares y laborales.

Fuente: Elaboración propia con base en la información de las entrevistas practicadas a la población.

En este sentido, el Código Sustantivo de Trabajo-CST (art. 127, 2019) establece que "todo trabajo dependiente debe ser remunerado", lo cual significa que la labor desempeñada cumple con las formalidades de una relación contractual y por lo tanto dichos trabajadores tienen derecho al reconocimiento de todas sus acreencias laborales, tal como se infiere de la norma laboral. "Constituye salario no solo la remuneración ordinaria fija o variable, sino todo lo que recibe el trabajador en dinero o en especie como contraprestación directa 
del servicio, sea cualquiera la forma o denominación que se adopte (...)" (Pedraza, Gamboa y Molina, 2015, p. 829). Se infiere entonces del supuesto normativo que se trata de la prestación de un servicio subordinado dependiente pues en virtud de su funcionalidad y características propias del trabajo, no permite acceder a otra opción laboral de tiempo, modo y lugar. Es así que la dependencia, en palabras de Manrique (2013), ha sido esencial en el desarrollo del derecho laboral.

En el marco del contrato de trabajo, trabajador y empleador acuerdan otorgar a este ultimo la facultad jurídica para dirigir, reglamentar y disciplinar el trabajo objeto de la prestación, en tanto que el trabajador se inserta en la empresa, formando parte de un proceso dirigido por el empleador para alcanzar unos fines determinados (...)” En el ordenamiento jurídico colombiano, la definición legal del trabajo subordinado o dependiente aparece en el artículo 23.1, literal b del CST, como la facultad del empleador para exigirle al trabajador, el cumplimiento de órdenes, en cualquier momento, en cuanto al modo, tiempo o cantidad de trabajo e imponerle reglamentos (pp. 65-66).

De esta forma, la subordinación o dependencia se evidencia en la población intervenida como elemento central en la relación contractual surgida entre las partes. Respecto al salario, según nos muestra la Figura 3, un alto porcentaje los trabajadores lo perciben cada quince días, y en orden descendente pero cercano al rango mayoritario lo perciben de forma semanal y en menos rango, mensualmente. Se deduce entonces, que una razón por la cual el salario es más habitual, es por la condición personal de este tipo de población agrícola, que como ya se enunció anteriormente la mayoría son mujeres cabeza de familia. Por lo tanto, el pago del salario se constituye como un elemento esencial en su desarrollo vital y cuotidiano. Tal como se desprende del CST (art. 132, 2019), "las partes pueden pactar libremente la modalidad de salario", sin desconocer el sentido jurídico de la norma respecto a los demás derechos que corresponden como por ejemplo el reconocimiento de las 
prestaciones sociales ${ }^{2}$. Desde esta lógica y como es natural el salario y su pago habitual constituye la base del sostenimiento de toda la población, pero no así, el reconocimiento de las prestaciones sociales. Se observa mayoritariamente que el salario es utilizado para el sostenimiento del hogar, educación, alimentación, servicios etc., y muy poco porcentaje lo destinan para otros gastos.

Ahora bien, respecto a la jornada de trabajo, la Figura 3 indica como el tiempo laborado desborda el límite ordinario permitido, es decir, 8 horas diarias y 48 horas semanales, tal como se estipula desde los artículos 158 al 171 del CST(2019), referentes a la modulación de la jornada de trabajo, notándose también que no hay reconocimiento de horas extras de ningún tipo, pues se colige que el objetivo del trabajador es entregar el pedido a toda costa, pues además este varia respecto a la cantidad solicitada. Es decir, se puede establecer el principio de proporcionalidad "a más pedido y a clima lluvioso también más tiempo empleado en sacar el producto del predio y conducirlo a las bodegas donde finalmente se organiza por las trabajadoras para concretar el envío" CST(2019).

Dicha situación implica que de forma general no se puede establecer un horario fijo para la ejecución de la labor, pues este depende de condiciones externas que lo hacen variar. Sin embargo, como lo entiende (Álvares et al., 2013), "que pese al límite establecido por la noción de la jornada ordinaria sea la convenida por las partes o la máxima legal, la ley prevé la posibilidad de trabajo en horas que superan dicho límite y lo califica de trabajo suplementario o de horas extras o jornada extraordinaria" (p. 389). Lo cierto es,

\footnotetext{
${ }^{2}$ En este mismo sentido y desde el contexto de los artículos 127 y 128 del CST (2019), estipulan lo que debe entenderse como salario y para tal fin señalan que a). El elemento esencial del salario es de un pago el cual constituye la contraprestación directa del servicio. b). Desde la perspectiva económica el salario es el precio que se reconoce por la mercancía trabajo. c). Para que se den las condiciones de salario debe haber prestación de servicio de forma directa. d). Conexión directa entre trabajo y salario. Aspectos que, son naturales de la relación laboral y con fundamento en el supuesto normativo y en el contrato, tal como lo define Álvarez, Arango, Baena, Barrera, Blanco, Borda, Uribe y Wilches, 2013, p. 357, son naturales de la relación laboral y con fundamento en el supuesto normativo y en el contrato.
} 
que el trabajador excede las 8 horas diarias y 48 semanales sin tener ningún reconocimiento de horas extras lo cual no garantiza lo contenido en la norma y lo estipulado por la OIT referente a las prerrogativas de derechos laborales y menos aún desde el contexto de trabajo decente.

Por otra parte, la mayoría de la población intervenida lleva laborando en las empresas comercializadoras de cebolla larga de Aquitania-Boyacá, más de 11 años, y en un rango un poco menor, hasta 11 años lo cual indica continuidad y permanencia en el tiempo lo que garantiza aún más el reconocimiento de sus prestaciones sociales. En materia de cotización a pensión la mayoría de los trabajadores intervenidos no han cotizado, pero si llevan trabajando entre $11 \mathrm{y}$ 20 años en dichas empresas, condición negativa respecto al tiempo, pues a medida que pasan los años al trabajador ya le será más difícil acceder a una pensión, en primer lugar, por el salario tan bajo que no alcanza para cubrir el porcentaje debido y en segunda instancia por la edad de los trabajadores que como se analizó, la mayoría oscilan entre 30 y 60 años en adelante, los cuales llevan mucho tiempo laborando en estas empresas y nunca han cotizado al sistema lo cual crea en el imaginario de los trabajadores lo "absurdo" de cotizar a esta altura de los años.

Desde esta óptica, y según Gamboa, (2017), el contenido normativo de la Ley 100 (art. 10, 1993), establece que "el objeto del Sistema General de Pensiones, es garantizar a la población el amparo contra las contingencias derivadas de la vejez, invalidez, muerte, mediante el reconocimiento de las pensiones y prestaciones que determina la ley (...)", (p. 3). Además de este derecho otorgado a los trabajadores, la Constitución Política (art. 48, 1991) establece la irrenunciabilidad del derecho a la seguridad social y entre ellos la pensión. De igual forma, se puede establecer que el "derecho pensional es una especie dentro de la generalidad del derecho fundamental a la seguridad social y por eso goza del amparo establecido por el artículo 86 de la Carta Magna” (Sentencia T- 471, 1992).

Desde este margen de garantías dado por nuestro ordenamiento jurídico se puede constatar la ineficacia normativa en los trabaja- 
dores objeto de la unidad de análisis, pues como se desprende de la Figura 3, ellos cumplen las exigencias del precepto para el reconocimiento de todas sus prestaciones sociales. Sin embargo, en la realidad no se evidencia y por ende se deriva como consecuencia, la vulneración de sus derechos fundamentales y el detrimento en cuanto al alcance del contenido de lo preceptuado como "trabajo decente".

Este tercer y último aspecto reflejado en la Figura 4, muestra que la mayoría de los trabajadores del sector agrícola objeto de la investigación laboran solamente en las empresas de cebolla larga, sin tener la posibilidad de realizar otra labor, es decir cumplen con las debidas formalidades que configuran un contrato laboral y por ende la protección de los derechos prestacionales que nacen de dicha relación. Realidad de la que están enterados un alto porcentaje de los entrevistados.

Aplicabilidad de las prerrogativas establecidas en los planteamientos de la OIT, relacionados con el trabajo decente respecto al trabajo desarrollado en este sector agricola de Aquitania - Boyacá.

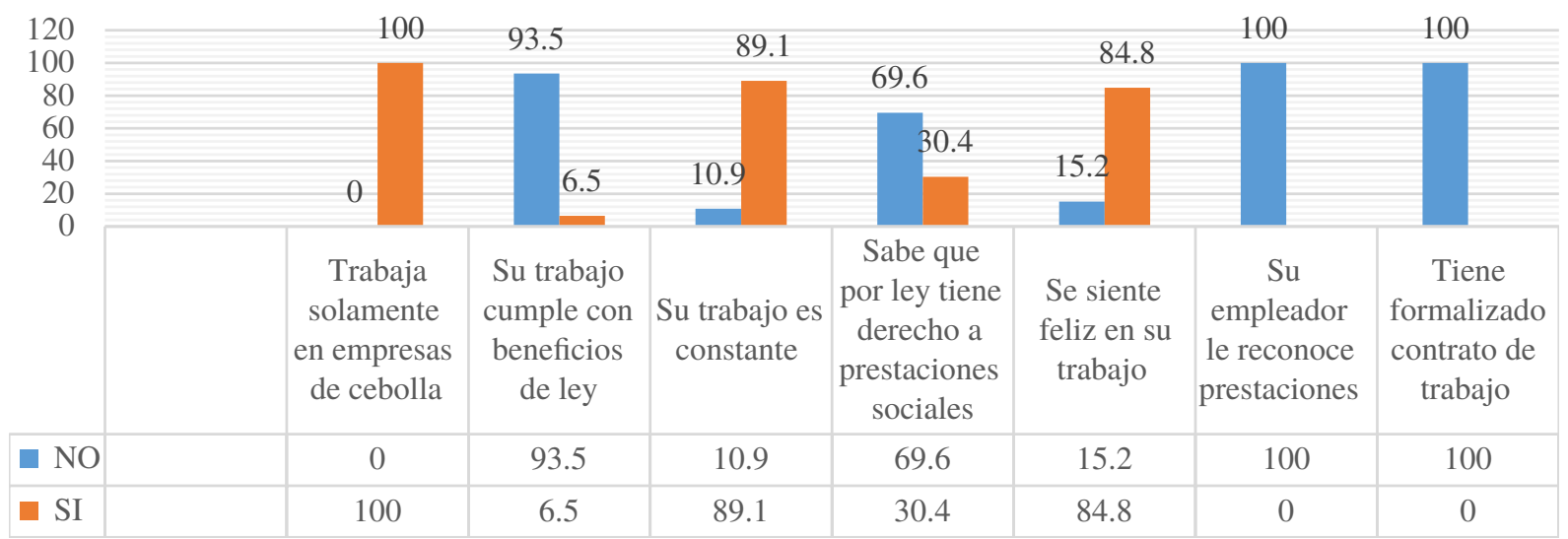

Figura 4. Empleador y condiciones de empleo. Fuente: Elaboración propia con base en la información de las entrevistas practicadas a la población. 
En este contexto, emergen principios esenciales propios del ámbito laboral que, según Manrique (2013), se sintetizan de la siguiente forma:

Principio protector: el cual se manifiesta en a. Indubio pro operario; b. regla de la aplicación de la norma más favorable; c. Regla de la condición más beneficiosa. Además, los principios de la irrenunciabilidad de los derechos, primacía de la realidad, de la razonabilidad y de la buena fe. Estos principios se encuentran dispersos a lo largo de la Constitución como en el artículo 13, 25,26,39,55, 56 y de forma explícita en el artículo 53 (p. 90).

De esta manera, se hace aún más factible el reconocimiento de prestaciones sociales en virtud del contenido normativo y de la realidad sobre las formas. Una característica que evidencia la gráfica 4, es la constancia o permanencia que puede tener un trabajador en las empresas, por lo tanto, pensar en la exigencia a su empleador del pago de sus prestaciones legales implicará para el trabajador ser despedido por lo cual prefieren seguir laborando sin el reconocimiento de las mismas.

El total de la muestra poblacional, refleja que no tiene un contrato debidamente formalizado, es decir, un contrato escrito y adaptado a las exigencias normativas laborales. Tampoco recibe por la realización de su labor ningún tipo de prestación social. Aunque la mayoría son conscientes de sus derechos laborales, no se aventuran a una reclamación por vía jurídica, pues prefieren mantener sus puestos antes de entrar en cualquier pleito.

En este contexto, los derechos que nacen de la relación contractual en materia laboral se desvirtúan en razona que no se reconocen y tampoco se exigen. Por tanto, la seguridad social, elemento propio del Estado Social de Derecho-desde esta óptica se ve afectada en cuanto a su aplicación, pues al concebirse como un "aspecto facultativo", lo que se sigue es la vulneración de este tipo de derechos con consecuencias funestas para el trabajador y para el sistema. La Seguridad social, tal como lo define ya la Conferencia Internacional del Trabajo en 1944, reunida en Fila- 
delfia "engloba el conjunto de medidas adoptadas por la sociedad con el fin de garantizar a sus miembros, por medio de una organización apropiada, protección suficiente contra ciertos riesgos a los cuales se hallan expuestos" (Rodríguez, 2015, p. 36). Desde esta lógica, dichos trabajadores se verán abocados a carecer de una pensión, percibir solo el salario establecido por las partes, estar expuestos a asumir de forma personal cualquier riesgo con ocasión del desarrollo de su labor y carentes de cualquier otro servicio complementario.

Por otra parte, nuestro ordenamiento interno ha establecido un ámbito de protección constitucional en materia de seguridad social, sobre todo en el artículo 48 de la Carta Magna, el cual establece que "es un derecho irrenunciable para todos los habitantes del territorio, es un servicio público de carácter obligatorio, sobre la base de principios rectores como la eficiencia, universalidad y solidaridad". De igual forma la corte constitucional ha establecido que la seguridad social es un derecho fundamental ya sea en relación con la salud, pensiones o en general. Dicho concepto se puede visualizar en las sentencias T-760 (2008), SU-062 (2010), T-032 (2012), T- 164 (2013), entre otras.

Desde la perspectiva de la OIT, en el documento base del año 2008, sobre medición del trabajo decente establece la integralidad que debe tener todo programa relativo a dicho aspecto y sobre todo desde la normativa interna garantizar la cobertura de una seguridad social apropiada y adaptada a las condiciones particulares de cada sector laboral. Es así que, desde esta cosmovisión de protección y cobertura integral, la teleología jurídica laboral conduce a beneficiar a todo tipo de trabajador garantizándole el acceso a sus prestaciones sociales correspondientes y en esta medida la disminución de la marginalidad y la pobreza.

El Programa de Trabajo Decente se ocupa también de las condiciones de vida de los trabajadores y sus familias y no sólo del trabajo productivo y remunerado y del lugar de trabajo. Este interés está profundamente arraigado en la historia de la OIT. 
En la Declaración de Filadelfia los mandantes afirmaron que «la pobreza, en cualquier lugar, constituye un peligro para la prosperidad de todos", y encomendaron a la OIT el cometido de fomentar programas que permitan la elevación del nivel de vida. Entre los objetivos que se mencionan explícitamente figuran los siguientes: extender las medidas de seguridad social para garantizar ingresos básicos a quienes los necesiten y prestar asistencia médica completa; proteger a la infancia y a la maternidad; suministrar alimentos, vivienda y medios de recreo y cultura adecuados; y garantizar iguales oportunidades educativas y profesionales (OIT, 2008, p. 9).

En síntesis, tanto la OIT como el ordenamiento jurídico del Estado Social de Derecho colombiano, establecen pautas y lineamientos para que la norma en materia laboral sea efectiva en su aplicabilidad, sin embargo, notamos que dicha acción no se evidencia en la población objeto de análisis, ya sea por razones de desconocimiento de las disposiciones, flexibilización laboral, deficiencia en la exigencia normativa por parte de las instituciones jurídicas y temor por parte del trabajador a inmiscuirse en un proceso por los costos.

Ahora bien, la Figura 4, demuestra que en un alto porcentaje de la población analizada no ha recibido capacitación en materia de riesgos laborales y menos aún de la implementación de los elementos necesarios para el desempeño de la labor, lo cual acarrea una mayor posibilidad de sufrir algún tipo de accidente o calamidad. No obstante, la Ley 1562 (2012) antes enunciada en el artículo 1, prevé que el Sistema General de Riesgos Laborales “(...) esta destinado a prevenir, proteger y atender a los trabajadores de los efectos de las enfermedades y los accidentes que puedan ocurrirles con ocasión o como consecuencia del trabajo que desarrollan". 


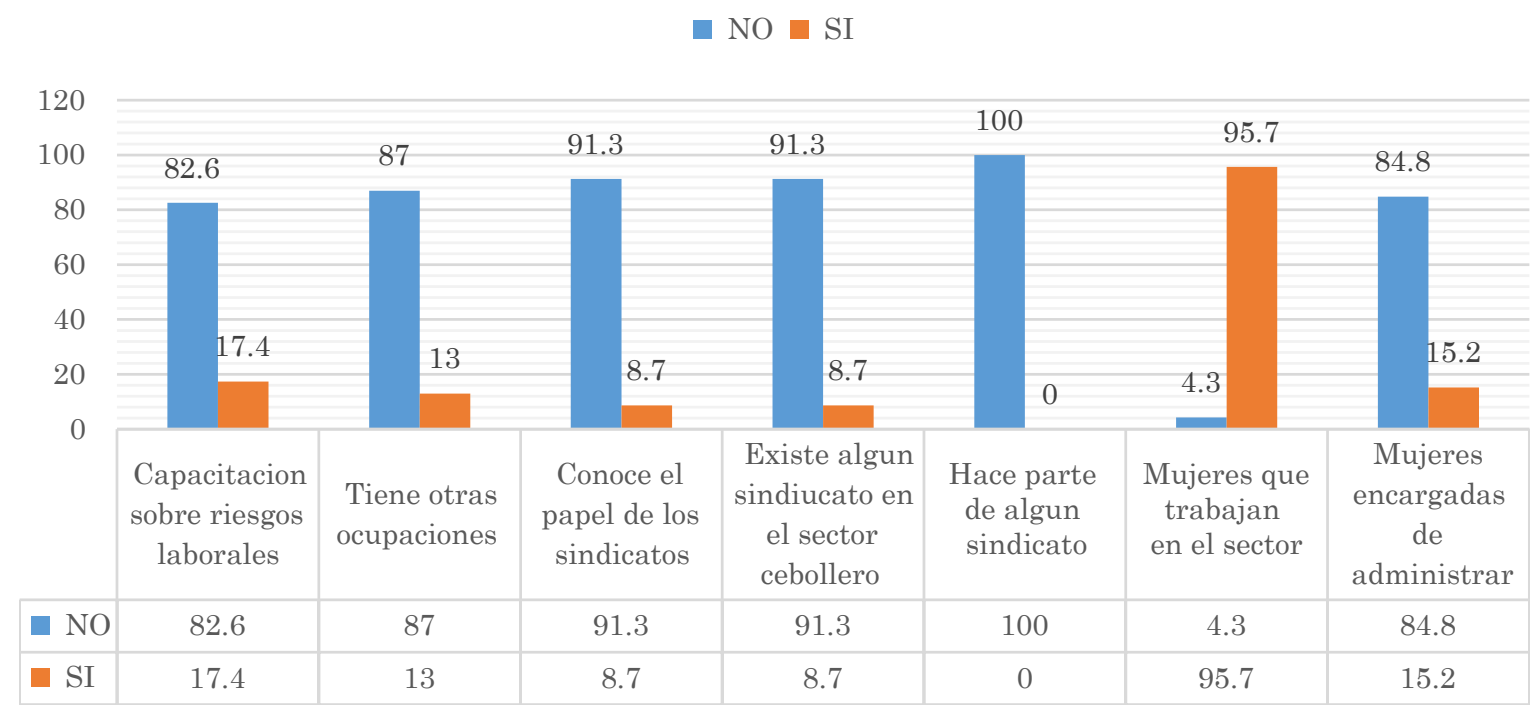

Figura 5. Empleador y condiciones de empleo: continuación de la figura 4. Fuente: Elaboración propia con base en la información de las entrevistas practicadas a la población.

Así mismo, la disposición citada, establece que todo aquello relacionado con salud ocupacional tendiente a la prevención de accidentes o enfermedades laborales hacen parte integrante del Sistema General de Riesgos Laborales. En este mismo sentido, la Resolución 0312 (2019) establece unos Estándares mínimos correspondientes a normas y procedimientos que deben tener en cuenta los empleadores con el fin de constatar la eficiente funcionalidad de la empresa en todo aspecto. Quiere decir que jurídicamente se exige el cumplimiento de ítems de operatividad, calidad y protección del trabajador ante cualquier calamidad o evento de origen laboral. Empero, dicha Resolución en el parágrafo 2 del artículo 2 , referente al campo de aplicación establece que "no están obligados a implementar los Estándares Mínimos establecidos en la presente Resolución, los trabajadores independientes con afiliación voluntaria al Sistema General de Riesgos Laborales de que trata de forma genérica el Decreto 1072 de 2015, Único Reglamentario del Sector Trabajo".

Significa pues, que la mayoría de los trabajadores del sector agrícola cebollero de Aquitania-Boyacá, al no estar afiliados por sus 
empleadores y aún menos de forma voluntaria, no son objeto de ésta regulación y por ende de las exigencias en cuestión de seguridad y salud en el trabajo, lo cual permite pensar en la precariedad laboral en este sector.

Por otra parte, referirse al derecho de asociación sindical en este sector laboral, es inoficioso en cuanto que dichas empresas comercializadoras de cebolla larga, apenas les otorgan a sus trabajadores un salario de acuerdo a su rendimiento, quedando en vilo las demás prestaciones. Situación tal que se refleja en la gráfica 4, pues la mayoría desconoce la función de los sindicatos y por razón lógica no hay posibilidad para estar vinculados a alguno que respalde sus derechos fundamentales.

La Constitución Política en el artículo 39 establece el derecho de sindicalización y asociación que tienen trabajadores y empleadores en el marco de los derechos fundamentales. Ospina (2013) afirma que el "derecho de asociación sindical en la Constitución Política está consagrado con los mismos alcances que tiene ese derecho en los Convenios de la OIT, es decir el derecho a organizar sindicatos por parte de los trabajadores" (p. 72). En esencia el "marco jurídico en Colombia se encuentra en la Carta Magna (art. 39, 1991), el Código Sustantivo del Trabajo (art. 353, 2019) y los convenios de la OIT” (Quintero y González, 2017, p, 33). Con todo, aunque existan los presupuestos normativos si no hay voluntad en su aplicación, no causa ningún cambio social.

Referente a la participación de la mujer en este tipo de trabajo notamos en la Figura 4, que la mayoría son mujeres, pero muy pocas en cargos de dirección y manejo. Las razones de tal situación es por la versatilidad que se debe tener a la hora de seleccionar el producto para su empacado y además porque no conlleva exigencia de fuerza como si la exigen los cargos de dirección y administración. En este sentido no se puede predicar quizá una vulneración de los derechos de la mujer en condiciones de equidad e igualdad precisamente por las exigencias del desarrollo de la labor. Sin embargo, si se evidencia dicha desigualdad en materia de salario. 


\section{Conclusiones}

Los lineamientos de la OIT respecto a la implementación del "trabajo decente" por parte del Estado colombiano, deben reflejarse en el orden jurídico interno en virtud de la firma y la ratificación de los convenios formalizados por nuestro País en esta materia. Desde esta lógica, las disposiciones de orden laboral en su contenido hacen explicito el carácter protector de todo tipo de trabajadores respecto a sus derechos laborales, esto es, de carácter salarial y prestacional, lo cual asegura el "modus vivendi" de quienes desarrollan su labor en cualquier sector incluyendo el agrícola. Además, dicho carácter protector se constituye como un deber institucional y un derecho constitucional como se conceptúa en el artículo 25 de nuestro Estatuto Laboral. Debe entenderse entonces que cualquier acción distinta a lo contenido en la norma se entenderá como una vulneración de la misma y por tanto el surgimiento de la legitimación para acudir a la jurisdicción laboral en aras de solicitar la protección jurídica de los derechos laborales y así poder asegurar la garantía de un trabajo decente.

El trabajo en el sector rural afronta grandes retos como las altas tasas de informalidad, la desigualdad en el uso y acceso a la tierra, la desprotección estatal, la poca estabilidad, la mala remuneración y el desconocimiento de los derechos laborales.

El análisis de los indicadores del trabajo decente en la población objeto de estudio refleja un enorme déficit de criterios que permitan aseverar que la actividad desarrollada por los trabajadores del sector agrícola cebollero sea de aquellas que se desarrollan en condiciones de justicia remunerativa, equidad, libertad, seguridad y respeto por la dignidad humana, lo que se traduce en una vulneración de derechos fundamentales y legales en materia de trabajo.

Los empleos del sector rural que se generan en el entorno agrícola cebollero del municipio de Aquitania-Boyacá, son precarios, pues son desarrollados en un entorno inestable e inseguro, donde adolece de suficientes condiciones laborales, como ingresos bajos, 
carencia de un piso de protección a través de los sistemas de Seguridad Social, condiciones que no permiten al trabajador mejorar sus condiciones de existencia.

De la presente investigación se puede resaltar que la gran mayoría de indicadores de medición del trabajo decente, son ineficientes e inoperantes, ya que el ámbito laboral de este sector agrícola son de carácter informal en las que no media un contrato de trabajo, en ocasiones se percibe un salario inferior al salario mínimo, las jornadas de trabajo resultan excesivas y en muchos casos inexistentes, no tienen ningún piso de protección social, al no encontrarse afiliados a salud, pensión y riesgos laborales, quedando así, dependiendo del sistema subsidiado y además desfinanciando el sistema contributivo, lo que a futuro va a generar problemas sociales y económicos graves de orden estatal, pues cuando dicha población no esté en edad y condiciones de trabajar, el Estado tendrá que proporcionarles lo necesario para su subsistencia.

Los trabajadores participantes de la investigación son en su mayoría mujeres cabeza de hogar que por la extensa jornada a la que se ven sometidas no tienen la posibilidad de conjugar la vida familiar con la personal, además que, por su baja escolaridad, falta de capacitación, rango de edad y escasez de empleabilidad en la región se sumergen en una relación de dependencia económica respecto de su empleador lo que las convierte en una población vulnerable y susceptible de situaciones de acoso.

Si bien los indicadores muestran una estabilidad en el desarrollo de la actividad de prestación de servicios, ello se explica por las condiciones especiales de cada trabajador que fueron reseñadas anteriormente y la falta de otros medios de subsistencia en la región lo que los obliga a aceptar condiciones ilegales e injustas de empleo como las que se analizan.

En materia de derechos colectivos la precariedad es evidente ya que las tasas de sindicación son nulas, lo que impide el dialogo tripartito y la mejora de las condiciones de empleo a través de las organizaciones sindicales, imposibilitando así una relación labo- 
ral de carácter democrático, defensora y garante de los derechos humanos, sustituyéndola por una relación en la que la arbitrariedad y la vulneración de normas laborales es la regla.

Todos estos elementos nos permiten determinar que el trabajo decente en la realidad investigada, es inexistente debido a la dislocación entre las estipulaciones normativas tanto nacionales como internacionales que obligan en materia de trabajo al Estado colombiano a garantizar su protección. Así mismo, se puede determinar la capacidad de las normas de modificar la realidad del trabajo rural -en el que aún persisten condiciones laborales propias del siglo XIX-, a fin de otorgar a este sector una protección especial dado su carácter de desempeño. En este sentido, se puede inferir que falta incrementar aún más la importancia del valor del trabajo, del Sistema de Seguridad Social, de los servicios complementarios etc., los cuales motivan a establecer un contexto de trabajo decente.

Dado lo anterior, el contexto negativo de los trabajadores del sector agrícola, afecta la teleología y el alcance no solo los derechos laborales consagrados en nuestro ordenamiento jurídico, sino que también vulnera la política de trabajo decente trazada por el gobierno nacional y pone en peligro los objetivos de Desarrollo Sostenible de la Agenda 2030 de las Naciones Unidas.

De allí la necesidad que las autoridades nacionales formulen una política integral de protección y reivindicación de derechos de las poblaciones rurales que pase por la formalización laboral, la capacitación constante, el control minucioso por parte de los inspectores de trabajo y el acceso a una justicia laboral pronta y efectiva, con posibilidades reales de sindicación, negociación y huelga.

Solo así, se podrá reivindicar la condición humana desde su entorno antropológico, de la actividad productiva desde su entorno económico, rescatando la centralidad del trabajo humano como medio de realización personal y familiar que traerá como consecuencia seguramente un desarrollo social más justo, equitativo y humano, pero sobretodo racional y ético. 


\section{REFERENCIAS}

Álvarez, C., Arango, F., Baena, G., Barrera, M., Blanco, Ó., Borda, E., Uribe, V. (coord.) y Wilches, M. (coord.) (2013). Compendio Teórico Práctico de Derecho del Trabajo. Bogotá, D.C.: Legis.

Blanco, O. (2002). El concepto de Trabajo Decente: ¿un freno a la desregulación indiscriminada? Actualidad Laboral, (113), 10-15. Recuperado de http://legal.legis.com.co/document/ Index?obra=rlaboral\&document=rlaboral_7680752a7f0140 4ce0430a010151404c

Campos, R. D. (2017). Derecho laboral. (Ed. 8.) Bogotá, D.C.: Temis. CIT, (junio 10-21, 2019). 108.a reunion de la Conferencia Internacional del Trabajo. [Online]. Recuperado de https://www. ilo.org/ilc/ILCSessions/108/lang--es/index.htm

Cortes, G. J. (2016). Seguridad Social, derecho para todos. Bogotá, D.C.: Legis.

Gamboa, J. J. (2017). Régimen General de Pensiones. Bogotá, D.C.: Leyer.

Isaza, J. G. (2003). Flexibilización laboral: un análisis de sus efectos sociales para el caso colombiano. Equidad y Desarrollo, 1, 9-39. https://doi.org/10.19052/ed.398

Legis. (2018). Cartilla de Seguridad Social. Bogotá, D.C.: Legis.

Manrique, V. J. (2013). Introducción al concepto de derecho del trabajo y su vínculo con las formas de trabajo independiente, parasubordinado y autogestionado. Bogotá, D.C.: Externado.

OIT. (2008). Medición del trabajo decente. [TMEMDW/2008]. Ginebra: OIT. Recuperado de https://www.ilo.org/wcmsp5/ groups/public/---dgreports/---stat/documents/meetingdocument/wcms_100337.pdf

Ospina, D. E. (2013). Derecho Colectivo del Trabajo. Bogotá, D.C.: Ibáñez.

Ostau de Lafont de León, F. (2015). Derecho Internacional Laboral. Bogotá, D.C.: Universidad Católica de Colombia. 
Pedraza, A, Gamboa, J, y Molina, B. (2015). Código Laboral Sustantivo del Trabajo y de Procedimiento Laboral. Bogotá, D.C.: Leyer.

Puyana, S. A. (2013). El sistema General de Riesgos laborales en Colombia. Bogotá, D.C.: U. Externado de Colombia.

Quintero, L. J y González, H. E. (2017). Lecciones de derecho colectivo del trabajo. Bogotá, D.C.: Doctrina y Ley.

República de Colombia. Asamblea Nacional Constituyente. (1991). [Constitución política de Colombia]. Gaceta Constitucional, 1(114).

República de Colombia. Congreso de la República. (11 de julio de 2012). [Ley 1562]. Diario Oficial No. 48.488.

República de Colombia. Congreso de la República. (29 de diciembre de 2010) [Ley 1429]. Diario Oficial No. 47.937.

República de Colombia. Congreso de la República. (27 de diciembre de 2002). [Ley 789]. Diario Oficial No 45.046.

República de Colombia. Congreso de la República. (23 de diciembre de 1993). Art. 212. [Ley 100]. Diario Oficial No. 41.148.

República de Colombia. Congreso de la República. (28 de diciembre de 1990). [Ley 50]. Diario Oficial No. 39.618.

República de Colombia. Ministerio del Trabajo. (07 de junio de 1951). Codigo Sustantivo del Trabajo. [Actualización diciembre 2019]. Diario Oficial No. 27.622.

República de Colombia. Corte Constitucional. (22 de marzo de 2013). Exp. T- 3.728.593. [Sentencia T-164]. MP: Jorge Ignacio Pretelt Chaljub.

República de Colombia. Corte Constitucional. (1 de febrero de 2012). Exp. T-3.257.055. [Sentencia T-032]. MP: Jorge Ignacio Pretelt Chaljub.

República de Colombia. Corte Constitucional. (3 de febrero de 2010). Exp. T-2021850. [Sentencia SU-062]. MP: Humberto Antonio Sierra Porto.

República de Colombia. Corte Constitucional. (31 de julio de 2008). Exp. T-1281247 y acum. [Sentencia T-760]. MP: Manuel José Cepeda Espinosa. 
República de Colombia. Corte Constitucional. (17 de julio de 1992). Proceso de tutela No. 1449. [Sentencia T- 471]. MP: Jaime Sanin Greiffenstein.

República de Colombia. Ministerio del Tranajo. (13 de febrero de 2019). [Resolución 0312]. Diario Oficial No. 50.872.

República de Colombia. Ministerio del Tranajo. (26 de mayo de 2015). Único Reglamentario del Sector Trabajo. [Decreto 1072]. Diario Oficial No. 49.523.

Rodríguez, M. R. (2015). Estudios sobre seguridad social. Bogotá, D.C.: Ibáñez.

Rodríguez, C. (2007). ¿Competir con el empleo precario? Economía Colombiana, 320, 50-63,

Rojas, C. (2016). La flexibilización laboral en Colombia. Juridicas CUC, 12(1), 17-29. http://dx.doi.org/10.17981/juridcuc.12.1.2016.2

Romero, L. (2011). Armonización de las normas constitucionales, laborales y concursales frente al despido, objetivo por crisis empresarial a consecuencia de la globalización. Jurídicas CUC, 7(1), 281-298. Recuperado de https://revistascientificas.cuc.edu.co/juridicascuc/article/view/428

Roth, U. (2004). El Cambio Social Comunitario: Análisis de la Influencia de los factores de Implantación y Asimilación sobre la Aceptación de las Innovaciones en contextos Comunitarios de Bolivia. Ajayu Órgano de Difusión Científica del Departamento de Psicología UCBSP, 2(2), 35-56. Recuperado de http://www.scielo.org.bo/pdf/rap/v2n2/v2n2a4. pdf

Solano, E. (2019). Estabilidad ocupacional reforzada en Colombia: una mirada crítica desde la jurisprudencia. Jurídicas CUC, 15(1). 47-68. http://dx.doi.org/10.17981/ juridcuc.15.1.2019.02

Villegas, A. J. (2016). Derecho administrativo laboral. (11 a ed.). Bogotá, D.C.: Legis. 
${ }^{1}$ Artículo de investigación científica que forma parte de los resultados de la investigación titulada "Trabajo decente desde los lineamientos de la Organización Internacional del Trabajo: su aplicabilidad en el sector agrícola cebollero de Aquitania-Boyacá, llevada a cabo en la Universidad de Boyacá dentro del grupo de investigación socio jurídica.

Hugo Armando Ramírez Acevedo es Doctorando en Derecho de la Universidad Castilla y La Mancha (España). Magister en Derecho Público de la Universidad Complutense de Madrid (España). Especialista en Derecho Laboral y Seguridad Social de la Universidad La Gran Colombia (Colombia) y Abogado de la misma institución. Licenciado en Filosofía, pensamiento político y económico de la Universidad Santo Tomás (Colombia). Licenciado en Teología de la Universidad Javeriana (Colombia). Docente de tiempo completo en la Uniboyacá (Colombia). Grupo de investigación Socio jurídica de la Línea de investigación en derecho público. https://orcid.org/00000001-6200-585X

Néstor Enrique Ulloa Corredor es Abogado de la U.P.T.C. Candidato a magister en derecho laboral de la Universidad Externado (Colombia). Especialista en Derecho Laboral y Seguridad Social. Especialista en Derecho Contractual de la Universidad del Rosario y docente de tiempo completo en la Universidad de Boyacá (Colombia). Grupo de investigación Socio jurídica de la Línea de investigación en derecho laboral. https://orcid.org/0000-0002-8845-2997 